Werner Kallmeyer

\title{
Vom Nutzen des technologischen Wandels in der Sprachwissenschaft: Gesprächsanalyse und automatische Sprachverarbeitung
}

"I've got a piece of data « - mit dieser Äußerung konnte zumindest in der ersten Hälfte der 70er Jahre ein Konversationsanalytiker Kollegen in eine »data session« einladen, d.h. eine Arbeitsgruppensitzung, die das Ziel hatte, ein Stück Transkript (meistens ohne dazugehörige Tonaufnahme) intensiv und bis in kleinste Details daraufhin zu betrachten, welche Ordnungsstrukturen und Verfahren der Herstellung dieser Ordnung im Gespräch aus dem Material selbst zu erkennen seien.

Die konversationsanalytische Zuwendung zur Realität der Sprachverwendung in der verbalen Interaktion, deren durchschlagender Erfolg mit ihrer mikroskopischen Perspektive und der Aufdeckung der Geordnetheit des scheinbar Ungeordneten bis in winzige Gesprächsdetails zusammenhing, wurde einerseits durch die technologische Entwicklung begünstigt und andererseits durch eine spezifische wissenschaftliche Orientierung in der phänomenologischen Soziologie.

Mit der Rezeption der Konversationsanalyse ist die Sprachwissenschaft der Umsetzung schon früh in programmatischer Weise formulierter Einsichten in ein konkretes Untersuchungsprogramm erheblich nähergekommen. So sind die beiden Kernstücke der inzwischen etablierten Gegenstandsdefinition früh vorformuliert worden: die Interaktivität in der Vorstellung vom grundlegenden Dualismus in der Sprache, deren Möglichkeit Anrede und Erwiderung bedingt, bei Wilhelm von Humboldt (1827) und die Zeitlichkeit und Prozeßhaftigkeit der Äußerungsproduktion in der Forderung nach einer Beschäftigung mit fortlaufender mündlicher Rede (vgl. Raumer 1857).

Die eigentümliche Haltung der Linguistik gegenüber der Sprachwirklichkeit und hier insbesondere der Rolle von mündlicher und geschriebener Sprache bzw. des Sprechens und Schreibens ist in den letzten Jahren präzise rekonstruiert und reflektiert worden. Klein (1985) spricht von einer paradoxen Haltung, die einerseits die primäre Stellung der gesprochenen Sprache bzw. des Sprechens gegenüber dem Schreiben betont und andererseits immer wieder zur dominanten Beschäftigung mit geschriebener Sprache zurückkehrt. Auer (1993) hat im Detail dargestellt, daß die Hinwendung zur geschriebenen Sprache als dem entscheidenden Gegenstand der Linguistik teilweise technologisch bedingt, teilweise aber in der Gegenstandskonstitution der Sprachwissenschaft verankert ist. Diese erfolgte zu einer Zeit, als die für 
die Untersuchung gesprochener Sprache erforderliche Technik noch nicht existierte. In der Folge, als die Technik vorhanden war, hatte sich unter dem Einfluß von Saussure und den Folgeentwicklungen des Strukturalismus ein anderes, schriftorientiertes Sprachmodell so fest etabliert, daß es eines regelrechten Paradigmenwechsels bedurfte (der "pragmatischen Wende«), um die Augen wieder zu öffnen für den primären Gegenstand.

Wichtige technische Voraussetzungen für die Untersuchung der gesprochenen Sprache im Stil der Gesprächsanalyse sind mit der Lösung von Problemen der Speicherung und des Zugriffs gegeben. Das dominante Problem war zunächst die Speicherung als Überwindung der Flüchtigkeit des Schalls. Seit über einhundert Jahren gibt es technische Mittel der Speicherung gesprochener Sprache, zunächst noch sehr beschränkt in der Kapazität und schwerfällig in der Handhabung, aber nach mehreren Entwicklungsschritten ist ein $\mathrm{Zu}$ stand erreicht, der das Aufnehmen und Wiedergeben von gesprochener Sprache jedermann relativ problemlos ermöglicht, ähnlich wie das Fotografieren. Die kleine, mobile und sehr leistungsfähige Aufnahmetechnik gestattet Sprachaufnahmen nahezu jederzeit und überall, soweit dies nicht aus ethischen Gründen unzulässig ist. Anders als noch zu Wenkers Zeiten mit einer ausschließlich durch Verschriftung speicherbaren gesprochenen Sprache ist heute kein ernsthaftes Problem, sich Tonbandbriefe zu schicken oder CDs mit Geburtstagsgrüßen zu besprechen.'

Für den Analysevorgang war die Wiederholungstaste nach dem Stenorettenprinzip ein Schlüsselelement. Die Wiederholungsfunktion gestattet, die Flüchtigkeit des Schalls gleichsam »abzubremsen« (vgl. Auer 1993). Gesteigert wird dieses Verfahren noch in Kombination mit einer »slow motion machine«, welche die Reduzierung der Wiedergabegeschwindigkeit ohne Absinken der Grundfrequenz erlaubt. Die Wiederholungsfunktion bezieht sich aber nur auf den Nahbereich. Springen in längeren Aufnahmen ist nach wie vor ein zeitaufwendiger Vorgang, zudem in der Regel mit Suchen beim Anhören verbunden. Hier hat die Digitalisierung mit der Möglichkeit, Zeitmarken zu setzen und den digitalisierten Schall mit einer Dateistruktur zu versehen, eine erhebliche Verbesserung gebracht. In einer Aufnahme von einer Stunde punktgenau bestimmte Stellen »anfahren«zu können, bringt mit dem Komfort sicher auch eine qualitative Verbesserung der Analysearbeit.

Die Untersuchung von Gesprächsdaten ist heute ein auf mehrere Disziplinen verteiltes und nach Ansätzen ausdifferenziertes Arbeitsgebiet, das sich mit ganz unterschiedlichen Fragestellungen befaßt (vgl. Kallmeyer, i. Vorb.). Und die »pieces of data «, die für exemplarische Analysen und sensibilisieren-

Im Prinzip war auch schon in den fünfziger Jahren für Privatleute möglich, eine Schallplatte pressen zu lassen, aber dieses Verfahren war doch an eine aufwendigere Technik gebunden und behielt einen Hauch von Exklusivität. 
des Eintauchen in die Fallstruktur benutzt werden, sind inzwischen Bestandteil von ganz beachtlichen Gesprächskorpora. Und nicht zuletzt: Es hat sich ein Forschungsstil entwickelt, der die in den frühen Jahren der Konversationsanalyse vorherrschende Orientierung auf die Transkriptanalyse relativiert zugunsten der unmittelbaren Wahrnehmung der Klanggestalt und ihrer Dynamik sowie in zunehmendem Maße auch der körperlichen Realität der sprachlichen Interaktion.

Nun gibt es inzwischen eine neue Entwicklung im Bereich der automatischen Sprachverarbeitung, welche die Rahmenbedingungen für die Beschäftigung mit gesprochener Sprache erheblich beeinflussen könnte. Gesprächsanalyse und automatische Sprachverarbeitung sind zwei völlig getrennte Wissenschaftswelten mit ganz unterschiedlichen Zielsetzungen, Gegenstandsbestimmungen und Methodologien. Neuerdings zeichnen sich aber gewisse Parallelitäten und Berührungspunkte ab, die es lohnend erscheinen lassen, das Verhältnis der beiden Arbeitsgebiete zu beleuchten. Anlaß dafür und Ausgangspunkt aller weiteren Überlegungen ist die Tatsache, daß die neuere automatische Sprachverarbeitung ein massives Interesse an dialogischer gesprochener Sprache entwickelt. Dieses Interesse geht auf die steigende Bedeutung der Spracherkennung und die Zukunftsaufgabe zurück, große multimediale Datenbanken einzurichten und zu nutzen. Nach den gegenwärtigen Projektionen werden große, unstrukturierte Datenmengen in Multimedia-Datenbanken für die Informationsgewinnung zu verarbeiten sein (vgl. u.a. Libermann 1991a). Eine wichtige Voraussetzung für die Lösung der Aufgaben werden Verfahren des automatischen Zugriffs auf natürliche gesprochene Sprache sein. Zu deren Entwicklung benötigt die maschinelle Sprachverarbeitung Sprachmodelle, welche die Eigenschaften gesprochener Sprache in ihren natürlichen Verwendungen angemessen erfassen. Die notwendige Grundlage dafür sind sehr große Korpora transkribierter Aufnahmen dialogisch gesprochener Sprache. In diesem Kontext bekommen statistisch-basierte Verfahren eine zentrale Rolle. Für deren sinnvollen Einsatz sind große Korpora erforderlich, sie können aber eben auch die Informationsmenge von großen Korpora analytisch nutzen.

Im Folgenden möchte ich mich mit einigen möglichen Implikationen dieser Entwicklung für die Erforschung der gesprochenen Sprache und der sprachlichen Interaktion befassen. Sie betreffen:

- Die langfristigen Strategien des Korpusaufbaus (1. und 2.).

- Den Zugang zur Sprachtechnologie, womit u.a. ein verbesserter Zugriff auf große Mengen von Gesprächsdaten verbunden ist (3.).

- Die methodische Entwicklung, insbesondere für die Analyse von Zusammenhängen, die mit den klassischen gesprächsanalytischen Verfahren nicht oder nur unvollkommen in den Griff zu bekommen sind (insbesondere großräumige Verteilungen) (4.). 


\section{Perspektiven des Aufbaus von Korpora gesprochener Sprache}

Die automatische Sprachverarbeitung benötigt Trainingsmaterial, das die Eigenschaften dialogisch gesprochener Sprache angemessen repräsentiert. Dazu gehören u.a. Sprecherwechsel, Überlappungen, »ungrammatische« Konstruktionen und die große Variabilität hinsichtlich unterschiedlicher Varietäten (Dialekt/Standard) und formaler bzw. informeller sprechsprachlicher Phänomene. Für die sog. Trainingskorpora sind sorgfältig von Hand entsprechend gesprächsanalytischen Standards transkribierte Korpora erforderlich (vgl. auch Thomson 1991, S. 174). Daraus ergibt sich u.U. für die Linguistik ein zusätzlicher Impuls, ihren Bestand an Gesprächskorpora zu sichten, zu verknüpfen und ausbauen. ${ }^{2}$

Gegenwärtig ist allgemein eine beschleunigte Entwicklung im Bereich der Korpustechnologie zu beobachten hin zum Aufbau und zur Handhabung großer Sprachkorpora. Als sehr große Korpora (VLLC, d.h. »very large linguistic corpora $\ll)$ gelten dabei Korpora mit mehr als $10^{7}$ laufenden Wörtern (vgl. u.a. Galle 1993). Motiviert wird die Orientierung auf sehr große Korpora durch die Erfahrung, daß kleine Korpora hinsichtlich seltener sprachlicher Phänomene kein realistisches Bild geben. Den größten Anteil an laufenden Wörtern eines Textes bildet eine relativ kleine Gruppe von sehr häufigen Wörtern, in erster Linie von sog. Funktionswörtern (Artikel, Päpositionen, Konjunktionen usw.), der größte Teil der verwendeten Wörter kommt in einem Text sehr selten, oft nur einmal vor und ein großer Teil der Wörter einer Sprache nicht in jedem Text (vgl. u.a Bergenholtz/Mugdan 1989). Man kann dieses Verhältnis etwas verbessern, wenn man spezifische Korpora für definierte Zwecke bildet, z.B. Korpora von Gebrauchsanweisungen für bestimmte technische Geräte oder von Mahnbriefen. Je allgemeiner jedoch ein Korpus sein soll und je mehr Variabilität an Textsorten, Themen, Sprachstilen usw. zugelassen werden, desto dringender wird eine exponentielle Steigerung des Korpusumfangs.

Es ist nicht ganz einfach, sich einen Überblick über den Stand der gegenwärtig verfügbaren Korpora zu verschaffen, weil die Informationen verstreut und viele Korpora ganz oder in Teilen nicht zugänglich sind. ${ }^{3}$

Daß unabhängig von den Interessen der automatischen Sprachverarbeitung die Sichtung und Konzentration von Sprachkorpora eine dringliche Aufgabe ist, speziell auch im Bereich der Korpora gesprochener Sprache, spiegelt sich u.a. darin, daß in den letzten Jahren vermehrt Anstrengungen zur Korpusdokumentationen erschein unternommen werden; vgl. z.B. Haas/Wagener 1992.

$3 \mathrm{Vgl}$. Allen 1989: "If it is not possible to access the data, there is no sense in mentioning it « - ganz so einfach kann man es sich vermutlich nicht machen angesichts der großen Grauzone von Korpora, die - noch - unter Verschluß gehalten werden. 
Publizierte Korpuslisten sind selektiv und teilweise auf spezifische Zwecke ausgerichtet und auf einen eingeschränkten Kreis von Beteiligten begrenzt. Aber auch auf dieser Grundlage werden die Zusammensetzung von bestimmten Korpustypen und ihre Größenordnung erkennbar. Ich beschränke mich hier auf die Seite der gesprochenen Sprache. Nicht zu übersehen ist, daß die insgesamt vorhandenen Computer-Korpora in erster Linie, oft ausschließlich geschriebene Sprache enthalten:

»Most corpora keep away from the problem of spoken language - with some honorable exceptions - and, for a corpus which in any way purports to reflect a state of the language<, this is most unfortunate. [...] In my own experience, there is no substitute for impromptu speech « (Sinclair 1991, 15/16).

Zunächst einmal hat die Technologieentwicklung gegen die gleichgewichtige Berücksichtigung gesprochener Sprache in den Computerkorpora gearbeitet. Der Grund dafür ist die unterschiedliche Verfügbarkeit von schriftlichen Texten und Verschriftlichungen von gesprochener Sprache. Computergespeicherte schriftliche Korpora sind inzwischen leicht in großem Umfang erweiterbar aufgrund der Tatsache, daß die Texte bereits in maschinenlesbarer Form auf Satzbändern o.ä. zur Verfügung stehen und nicht mehr wie in früheren Jahren neu am Bildschirm eingegeben werden müssen. Andere Texte, für die keine Satzbänder existieren, können gescannt werden. Die Folge ist, daß für schriftliche Sprache hinsichtlich der Menge keine prinzipiellen Beschränkungen mehr vorliegen. ${ }^{4}$

Gesprochene Sprache ist an sich in großer Menge problemlos verfügbar und seit der Entwicklung der Digitalisierungstechnologie sehr gut speicherbar und handhabbar. Sicher ist der Speicherplatz ein begrenztes Gut, aber es ist heute mit relativ einfachen Mitteln möglich, ein Nationalarchiv von Sprachaufnahmen aufzubauen. ${ }^{5}$ Die Medienanstalten (Rundfunk und Fernsehen) haben inzwischen Archive von Ton-/Video-Aufnahmen für ihre Sendungen und das Hintergrundmaterial oder richten derartige Archive ein, und die französische Nationalbibliothek besitzt ein zentrales Dokumentationszentrum, das alle in Frankreich ausgestrahlten Sprachsendungen speichert. Die neuen Verfahren der Digitalisierung, die u.a. auch Möglichkeiten der Datenkompression bieten ohne hörbaren Verlust, erleichtern eine ökonomische, nicht die Dimension des Praktikablen sprengende Archivierung. In kleinem Um-

4 So ist jedenfalls der Tenor der progressiven Korpustechnologen. Praktisch kann es dann doch sehr schwierig sein, u.a. wegen Fragen des Copyrights und der ökonomischen Interessen. Aber es ist sicher bezeichnend, daß bereits die Frage bedeutsam wird, wie die verfügbare Datenmasse durch Auswahl auf das Nützliche reduziert werden kann (vgl. Belica 1996 zur Vermeidung von Übersättigung durch uninteressante Texte).

5 Vgl. Mehnert (i. Vorb.) zu den Gründungen von Schallarchiven um die Jahrhundertwende in Wien, Paris, Berlin und St. Petersburg. 
fang existieren auch in den deutschen Universitäten Archive von Ton- und Fernsehaufnahmen, in der Regel mit medienwissenschaftlicher Orientierung. Das Problem ist die Transkription.

Die vorhandenen Korpora verschriftlicher gesprochener Sprache sind in unterschiedlichen Kontexten entstanden, und die Daten haben dementsprechend unterschiedliche Form:

- Zu computerlinguistischen Zwecken bzw. zu Zwecken der automatischen Spracherkennung zusammengestellte Korpora wie das Britisch National Corpus, mit einem kleineren Anteil gesprochener Sprache, Telefonkorpora der Industrie oder das Korpus mit Bahnauskünften und Terminabsprachen aus VERBMOBIL. Derartige Korpora sind in der Regel beschränkt hinsichtlich des Text-/Interaktionstyps und vereinfacht hinsichtlich des zugelassenen Niveaus von Phänomenen des natürlichen dialogischen Sprechens (vgl. auch Thomsen 1991, 174).

- Zu Zwecken der Sprachdokumentation aufgebaute Korpora, u.a. mit dialektologischer Orientierung: Beispiele sind das Lautarchiv an der Humboldt-Universität in Berlin (vgl. Mehnert i. Vorb.), das Deutsche Spracharchiv (Zwirner Archiv; vgl. Haas/Wagener 1992) oder der Language and Culture Atlas, dessen Korpus 6000 Stunden Fragen nach Fragebuch und freie Antworten umfaßt. ${ }^{6}$

- Gesprächsanalytische Korpora: Es exitiert eine beachtliche Menge an transkribiertem Gesprächsmaterial in verstreuten kleineren Korpora, die unterschiedlich spezifisch in der Zusammensetzung sind. Teilweise gibt es auch Ton- und Transkriptarchive mittleren Umfangs in den Universitäten als eine Art Arbeitsinstrument für die Studenten (z.B. in Bielefeld und Dortmund). Zum Teil werden diese Korpora für die computerlinguistische Auswertung aufbereitet, z.B. die Gesprächskorpora im Institut für deutsche Sprache in Mannheim (IDS). Eine verläßliche Schätzung, wieviel transkribiertes Gesprächsmaterial national oder international existiert, liegt nicht vor.

Ein Handicap für die Entwicklung von computerlesbaren Gesprächskorpora ist bzw. war zumindest das Zeilenblockformat: Die Sprecher werden wie die Instrumentenstimmen in einer Partitur parallel geführt, sodaß die relative Anordnung der Stimmen zueinander gleichzeitiges Sprechen, Pausen und Sprecherwechsel angibt. Dafür sind in den letzten Jahren spezifische Editoren entwickelt worden. ${ }^{7}$ Diese Editoren halten die Transkripte als Textdateien.

$6 \mathrm{Vgl}$. Herzog/Baviskar/Kiefer/Neumann/Putschke/Sunshine/Weinreich (1992/1995). Zur Dokumentation der verstreuten Bestände von Sprachaufnahmen vgl. auch Bausch/Wagener (i. Vorb.).

7 So im deutschen Sprachraum SyncWriter für MacIntosh, HIAT-Dos oder DIDA für Unix; vgl. auch Kallmeyer 1993. 
Für die automatische Sprachverarbeitung sind Partitur-Transkripte in ein Datenbank-Format zu überführen. Für den Mannheimer Transkripteditor DIDA geschieht das zur Zeit für COSMAS (COrpus Storage and Maintenance System; vgl. al-Wadi 1994), das zu diesem Zweck speziell für die Verwaltung von Diskursdaten (Transkript und Tonsignal) erweitert wird (COSMAS II). Bei der Überführung in die Datenbank wird der Fluß der sprachlichen Symbole automatisch in kleine Segmente zerlegt, welche unter Bezug auf das Datenmodell klassifiziert und relativ zum Kontext positioniert werden. Für die Klassifikation wird das TEI-Kategoriensystem verwendet (Text Encoding Initiative; vgl. Sperberg-McQueen/Burnard 1994), das derzeit die größten Aussichten hat, sich als internationaler Standard durchzusetzen. Der TEIVorschlag ist bereits sehr ausdifferenziert und weiterhin für Ergänzungen offen, sofern für das jeweilige Datenmodell relevante Eigenschaften nicht erfaßt sind. Die IDS-Erfahrungen sind bislang positiv.

Angesichts der ungeheuer aufwendigen Beschaffung und Transkription von Gesprächsmaterial sind Austausch und Mehrfachnutzung der Daten an sich besonders dringend, ggf. auch eine Sammlung und Inkorporierung von Gesprächsdaten unterschiedlicher Herkunft in einem allgemein verfügbaren Korpus. Aus verschiedenen Gründen geschieht dies nur begrenzt und langsam. Einmal sind Gesprächsmaterialien, insbesondere solche aus nicht ohne weiteres zugänglichen Situationen, für deren Erschließung oft viel Zeit und Energie eingesetzt werden müssen, für die Eigentümer ein kostbares Gut, das sie - sofern die Datenschutzbestimmungen dies überhaupt gestatten - nur im Austausch mit Gegenleistungen weitergeben. Weiter wird die Zusammenführung der verstreut vorhandenen Gesprächskorpora durch die Varianz der Transkriptionsweisen erschwert. Die Unterschiedlichkeit der Transkriptionen ist einerseits durch Untersuchungsinteressen motiviert, welche die Erfassung teilweise unterschiedlicher Phänomene notwendig machen, weiter durch die Berücksichtigung verschiedener Adressatengruppen (Lesbarkeit ggf. auch für Nicht-Spezialisten) und schließlich durch historisch gewachsene Gewohnheit und Schulenbildung. Eine Standardisierung ist dringend zu wünschen, vermutlich wird sich aber keine schnelle Vereinheitlichung durchsetzen lassen. Wenn übereinstimmende Phänomene konsistent notiert werden, muß eine automatische Umkodierung möglich sein. Aber es ist fraglich, ob sich der Aufwand an Programmierung für die Überführung auf der Ebene von Textdateien zwischen den zahlreichen Transkriptionsvarianten lohnt. Zukunftsträchtiger erscheint eigentlich aus heutiger Sicht der Weg über ein allgemeines Transferformat wie TEI-konformes SGML (Standard Generalized Markup Language), das für die Aufnahme von Gesprächsdaten in Datenbanken und den Datenaustausch zu verwenden ist.

Gegenwärtig sehen die nationalen Referenzkorpora im Bereich der Computerlinguistik wie das British National Corpus und nach dessen Vorbild entstehende Korpora ca. 10\% des gesamten Korpus für transkribierte sprochene Sprache vor. Angesichts des schnellen Wachstums der Korpora schriftli- 
cher Texte bereitet es zur Zeit noch große Mühe, diesen Anteil von gesprochener Sprache zu erreichen. Doch unabhängig davon ist fraglich, ob die im Mengenverhältnis 9:1 implizierte Relevanzabstufung zwischen schriftlichen und mündlichen Texten für die automatische Sprachverarbeitung in Zukunft angemessen ist. Die Sprachindustrie hat erklärtermaßen Bedarf an Korpora gesprochener Sprache, die um mehrere Stufen oberhalb der gegenwärtigen Größenordnung liegen. Den Schätzungen für den Korpusaufbau liegen u.a. Überlegungen der folgenden Art zugrunde: Ein Mensch hört im Laufe eines Jahres etwa 20 Mio gesprocher Wörter; gemessen daran erscheint ein Korpus von ca. 100 Mio Wörtern eine vernünftige Größe (vgl. Liberman 1991a).

Die genannte Größenordnung von 100 Mio laufenden Wörtern gilt heute für schriftliche Korpora als eine Meßlatte für seriöse allgemeine (d.h. nicht textsorten- und themenspezifische) Korpora (vgl. z.B. das nationale britische Korpus). Der Korpusaufbau wird hier nicht stehenbleiben; man kann sich leicht vorstellen, welche Auswirkung die Einbeziehung einer großen Zahl von Textsorten auf den Bedarf an Textmenge hat. ${ }^{8}$ Aber für den Aufbau von Korpora gesprochener Sprache ist diese Größenordnung geradezu revolutionär: Sinclairs am Beginn der 60er Jahre zusammengestelltes Korpus gesprochener Sprache umfaßte 125.000 Wörter, das Freiburger Gesprächskorpus aus den 70er Jahren enthält 300.000 Wörter. Das IDS-Korpus transkribierter und maschinell gespeicherter Gesprächsaufnahmen umfaßt derzeit etwa 1.7 Mio Wörter (und kann damit gegenwärtig schon als groß gelten).

Für gesprächsanalytische Belange ist die reine Größe eines Korpus an sich relativ uninteressant, viel wichtiger ist eine überschaubare Sammlung von wirklich aussagekräftigen Fällen. Aber in dem Maße wie sich die gesprächsanalytischen Fragestellungen ausdifferenzieren und auch differentielle Gesichtspunkte (situative, rollengebundene Unterschiede usw.) einbezogen werden, wächst der Bedarf an größeren Vergleichsmengen von Gesprächsmaterial.

\section{Entwicklungen der Korpustheorie}

Die Konversationsanalyse hat Gesprächsdaten gesammelt, aber sie eigentlich nie als geschlossenes Korpus betrachtet (vgl. auch Auer 1993, S. 127). Das Interesse richtet sich auf Beispielmengen (»collections«), die im Kern nach dem Prinzip des »theoretischen Sampling« (Glaser/Strauss 1967; Strauss 1991 ) zusammengestellt werden: Sie sollen hinreichend typische Fälle, Varianten und Grenzfälle umfassen, so daß alle Kategorien für die Eigenschaften

8 Z.B. enthält das Trierer Korpus 68 Mio laufende Wörtern aus dpa-Texten von 1988-1989 und 35 Mio laufenden Wörtern aus taz-Artikeln von Mitte 1988 bis Ende 1991 (vgl. Galle 1993); das schriftliche Korpus des IDS umfaßt derzeit etwa 200 Mio laufende Wörter, bei einer relativ breiten Mischung von Textsorten. 
des Gegenstandes und ihre Beziehungen untereinander belegt sind. Ein geläufiges Verfahren für die Untersuchung sehr allgemeiner Regularitäten wie z.B. des Sprecherwechselmechanismus ist, eine Fallmenge von "glatten«, d.h. problemlosen und unauffälligen Interaktionsverläufen mit einer Menge von Fällen zu konfrontieren, in denen sich in Bezug auf die fragliche Regularität Turbulenzen zeigen (vgl. Sacks/Schegloff/Jefferson 1974). Die analytische Auflösung besteht im Nachweis, daß auch die Struktur der problematischen Fällen durch die Orientierung der Beteiligten auf die allgemeine Regularität zu erklären ist. ${ }^{9}$ Die Anforderungen an die Materialzusammenstellung ändern sich etwas mit der Betrachtung unterschiedlicher Kommunikationssituationen und Interaktionstypen und dem Einsatz der Gesprächsanalyse als Untersuchungsinstrument in ethnographischen und soziolinguistischen Untersuchungen. Hier werden Beispielmengen für die typischen Eigenschaften der Kommunikation unter unterschiedlichen Rahmenbedingungen zusaammengestellt und miteinander verglichen. Aber auch dabei spielen im engeren Sinne korpustheoretische Fragen im Sinne der klassischen Doktrin repräsentativer Korpora, wonach das Korpus als Stichprobe ein verkleinertes Abbild einer Grundgesamtheit bietet (Gleason 1955), keine zentrale Rolle.

Interessanterweise entwickeln sich gegenwärtig auch im Bereich der automatischen Sprachverarbeitung als Alternative zur klassischen Korpustheorie Auffassungen, die auf eine flexible, fragespezifische Zusammenstellung von Korpora hinauslaufen und Ansatzpunkte für Forschungsstrategien bieten, die dem »theoretischen Sampling« der qualitativen Forschung näherkommen.

Die vielfach als modellhaft angesehenen Referenzkorpora im Bereich der Computerlinguistik, die nach einem bestimmten Schlüssel zusammengestellt für eine Reihe von Sprachen bzw. Kommunikationsgemeinschaften übereinstimmen (vgl. u.a. Rieger 1979), basieren im Kern noch auf der Vorstellung von Korpora als fixen Textsammlungen, deren Zusammensetzung nach bestimmten Gesichtspunkten festgelegt ist in der Erwartung, z.B. alle Textformen, die für den öffentlichen Diskurs in einer Gemeinschaft relevant sind, in einem angemessenen Mengenverhältnis berücksichtigt zu haben. Repräsentativität wird dabei allerdings nur eingeschränkt beansprucht.

Die Debatte um die Repräsentativität von Sprachkorpora hat im Kern gezeigt, daß es nicht möglich ist, ein im strengen Sinne repräsentatives allgemeines Sprachkorpus zu bilden. Die geläufigen Argumente sind: Die Grundgesamtheit der Sprachverwendungen in einer Gesellschaft ist nicht wohldefiniert, u.a. weil über sie viel zu wenig bekannt ist; das Korpus muß auf die jeweiligen Analyseziele zugeschnitten werden. ${ }^{10}$ Auch die Längerfri-

\footnotetext{
9 So in allen "klassischen « Arbeiten der Konversationsanalyse wie in Sacks/Schegloff/Jefferson 1974; vgl. auch Kallmeyer 1988.

$10 \mathrm{Vgl}$. u.a. Bergenholtz/Mugdan 1989. Auch die Abmilderung der Repräsentativitätsanforderungen zur exemplarischen Qualität (Bungarten 1979) schafft nicht grundsätzlich Abhilfe.
} 
stigkeit der Festlegung von Korpuszusammensetzungen, so gut sie für den Moment und die jeweilige Gesellschaft begründet sein mögen, ist problematisch. Die Kommunikationsstruktur einer Gesellschaft ändert sich machmal sehr schnell und damit die Bedeutung bestimmter Kommunikationsformen. So wäre seit wenigen Jahren eine Vorstellung von der Kommunikationswirklichkeit in unserer Gesellschaft ohne die Einbeziehung des elektronischen Kommunikationsaustausches über das Internet unrealistisch und dementsprechend eine in den 80er Jahren für längere Dauer festgelegte Korpusstruktur heute nicht mehr angemessen.

Um derartige Probleme zu vermeiden, geht die Entwicklung der Korpustechnologie hin zu virtuellen Korpora, die aus der Gesamtmenge der zur Verfügung stehenden Korpora im Hinblick auf bestimmte Fragestellungen durch Auswahl und Verknüpfung zusammengestellt werden. Sie sind virtuell in dem Sinne, daß die Anlage des Grundkorpus bzw. der benutzten Grundkorpora nicht tangiert wird. Definiert ist das virtuelle Korpus über den Zugriff (vgl. Neumann 1996). Ein Anwendungsfall des Konzepts des virtuellen Korpus sind Monitorkorpora (vgl. a. Sinclair 1991). Derartige Korpora sind insbesondere zur Betrachtung von fortschreitenden Entwicklungen notwendig, z.B. für die Untersuchung von Neologismen im Sprachgebrauch. Ein Zeitfenster, z.B. von fünf Jahren oder zwei Monaten, wird über das zeitlich geordnete Korpus gelegt und schrittweise verschoben, so daß die Veränderungen jeweils relativ zu einer aktuellen Textmenge statistisch erfaßt werden können (vgl. a. Belica 1996).

Die Bewertung der Angemessenheit von fragespezifisch zusammengestellten virtuellen Korpora erfordert spezifische Verfahren, sofern man nicht die Schwierigkeiten der Repräsentativitätsdiskussion wiederholen will. Vorgeschlagen wird das interne Kriterium der "Sättigung «: Geeignet erscheinende, linguistisch definierte und begründete Eigenschaften der Texte werden statistisch berechnet hinsichtlich Signifikanz und Variation in einer Textmenge. Das Ausgangskorpus wird schrittweise erweitert, wobei die Berechnung jedesmal wiederholt wird. Wenn sich mit der Vergrößerung des Korpus keine Veränderung mehr ergibt, also das Verhalten des gewählten linguistischen Phänomens invariant ist, gilt das Korpus als gesättigt. (vgl. Belica 1996; Neumann 1996). Wenn man z.B. daran interessiert ist, Korpora von unterschiedlicher Formalität/Informalität zusammenzustellen, kann man u.a. als einen Indikator die Verb-Erst- oder -Zweitstellung nach Konjunktionen wie weil benutzen, ausgehend von der Hypothese, daß derartige Konstruktionen gleichsam einen Signalwert haben und sich andere Phänomene, die ebenfalls mit Formalität/Informalität zusammenhängen, ähnlich verhalten. Wenn für eine Gruppe von informellen Texten ein bestimmter statistischer Wert errechnet ist, der deutlich von der Verteilung im Gesamtkorpus abweicht, kann dieser Wert als ein Kriterium benutzt werden für die Zusammenstellung von Korpora informeller Texte; dabei wird dann das Verfahren der Sättigungsprüfung eingesetzt. Im konkreten Fall wird man kaum nur mit einem einfachen Kriterium wie der 
Verbstellung nach weil arbeiten, sondern eine Reihe von sprachlichen Phänomenen (wie bestimmte Interjektionen und Ellipsen) und die statistischen Relationen zwischen ihnen einbeziehen.

In einer Hinsicht bleibt das Problem der Zusammensetzung des Grundkorpus allerdings erhalten. Aus ganz pragmatischen Gründen wird der weitere Korpusaufbau im Bereich der automatischen Sprachverarbeitung von den leicht verfügbaren Datenbeständen ausgehen. Man muß sich aber bewußt bleiben, daß die Technologieentwicklung Ungleichgewichtigkeiten produziert: Die Verfügbarkeit von fast beliebig großen schriftlichen Korpora gilt z.B. nur für den Bereich der gedruckten oder für den Druck vorgesehenen schriftlichen Texte, nicht jedoch für andere Bereiche von Texten, die nicht für die Veröffentlichung produziert werden (vgl. a. Bergenholtz/Mugdan 1993). Für Korpora gesprochener Sprache kann man eine vergleichbare Warnung vor der einseitigen oder übermäßigen Berücksichtigung von Aufnahmen aus den öffentlichen Medien aussprechen. Es muß ein Gegengewicht gegen den Sog der leichten Verfügbarkeit großer Datenmengen geschaffen werden in Form von Sammlungsstrategien, welche sich auf andere, schwerer zugängliche Textformen konzentrieren.

Die Zusammenstellung und Definition von Korpora und Teilkorpora (wie auch die Anwendung von Rechercheverfahren) impliziert weitreichende Annahmen über Kommunikations- und Sprachstrukturen. Die umfangreiche Aufnahme von Zeitungstexten in allgemeine Korpora z.B. folgt einerseits pragmatischen Gründen, wird andererseits aber auch mit der Annahme begründet, daß die Zeitungstexte den gesellschaftlich relevanten Sprachgebrauch abbilden. Das gilt aber nur unter bestimmten politischen und kommunikationsstrukturellen Bedingungen, und sie wird fragwürdig, wenn die Zeitungstexte starken Regulierungen unterliegen (z.B. im Sinne von offiziellen, politisch kontrollierten Verlautbarungstexten - so geben die Texte in »Neues Deutschland « sicher nicht allgemein »die Sprache der DDR « wieder). Auch die ungleichgewichtige Zusammenstellung von Referenzkorpora mit ihrer geringen Menge an gesprochener Sprache folgt zwar einerseits pragmatischen Gesichtspunkten, stützt sich aber zugleich auf die implizite oder explizite Legitimation, daß die Ergebnisse aus der Untersuchung der schriftlichen Texte generalisierbar sind und auf die gesprochene Sprache weitgehend übertragen werden können unter Ergänzung um die Berücksichtigung von deren differentiellen Spezifika. Ob das eine geeignete Strategie ist, ist aber eine noch zu klärende linguistische Frage.

In der Konzeption der stabilen Referenzkorpora werden derartige Implikationen gleichsam einzementiert. Die Strukturierung von Referenzkorpora ist in der Regel Ergebnis eines komplizierten Aushandlungsprozesses und auf langfristige Stabilität angelegt. Die neue Flexibilität der Korpustechnologie weckt die Hoffnung, daß sie viel eher Spielraum und auch Anlaß geben wird zur eingehenderen Reflexion über die gesellschaftliche Kommunikationsstruktur einschließlich der Vernetzung von unterschiedlichen Kommunikati- 
onskreisen wie öffentlichen, halböffentlichen und privaten, mündlichen und schriftlichen Diskursen. Ganz allgemein müssen Texte/Gesprächsdokumente als Bestandteil eines Kommunikationszusammenhanges begriffen werden, der im Prinzip die Rezeption und Verarbeitung einschließt. ${ }^{11}$

Die linguistische Auseinandersetzung mit korpustheoretischen Konzepten und den Kriterien für die Angemessenheit von Auswahlkorpora ist geeignet, weiterführende Überlegungen anzustoßen. Man kann sich z.B. im Hinblick auf die zeitliche Gliederung eines Korpus für die Untersuchung von Sprachveränderungen nach der Aktualitätsdauer von Texten ${ }^{12}$ und ihrem Zusammenhang mit der zyklischen Organisation des Kommunikationsprozesses (täglich, wöchentlich usw.) fragen, nach den unterschiedlichen Bedingungen für schriftliche Texte und mündliche Kommunikation hinsichtlich der zyklischen Organisation und danach, wie mündliche Kommunikationsformen die zeitliche Streckung der Aktualitäts- oder Geltungsdauer organisieren. ${ }^{13}$ Sinclairs Empfehlung (1991) an den Linguisten, sich auf die Analyse der in einem Korpus erscheinenden sprachlichen Phänomene zu beschränken und zu seiner Entlastung die Zusammenstellung des Korpus getrost einem Sozialwissenschaftler zu überlassen, sollte man deshalb nicht folgen. Sie läuft darauf hinaus, einen fruchtbaren Arbeitszusammenhang zu zertrennen, wie er gerade in ethnographischen und soziolinguistischen Ansätzen realisiert wird.

Hinsichtlich der Auseinandersetzung mit der Einbettung von Ereignissen verbaler Interaktion in übergreifende sozial-kommunikative Prozesse hat sich auch die Praxis der Gesprächsanalyse gegenüber der klassischen Konversationsanalyse geändert. Diese verfolgte zunächst eine Dekontextualisierungsstrategie (»Don't contextualize«, so eine Anweisung von Harvey Sacks im Seminar). Dahinter stand die Annahme, daß die Materialausschnitte alles für die lokale Herstellung von sozialer Ordnung Relevante erkennbar enthalten

11 Neben der entwickelten Forschung zur Mündlichkeit hat sich inzwischen auch die Beschäftigung mit der Schriftlichkeit als zentraler kultureller Leistung etabliert (vgl. Goody 1986, Olson 1994, Günther/Ludwig (Hg.) 1996. Die Verbindung zwischen beiden, die "Schnittstelle « zwischen Mündlichkeit und Schriftlichkeit (Goody 1987), also die Rezeption und Weiterverarbeitung von schriftlichen Texten in mündlicher Kommunikation wie auch der Weg von mündlicher Kommunikation zu schriftlichen Texten ist erst in ersten Ansätzen Gegenstand der Forschung; Für einen Aspekt dieses Zusammenhanges, das gemeinsame Schreiben vgl. z.B. Dausendschön/Gülich/Krafft 1992. In diesen Kontext gehört auch die Reaktion auf Medienkommunikation in der weiteren alltagsweltlichen und institutionellen Kommunikation; für die Rezeption von Fernsehsendungen vgl. u.a. Holly/Püschel ( $\mathrm{Hg}$.) 1993.

12 Vgl. den Begriff der >Dispersivität $<$ von Texten im Sinne von Aktualitätsdauer in Belica 1996.

13 Solche Gesichtspunkte spielen in der ethnographischen Analyse von Kommunikationsgemeinschaften eine wesentliche Rolle. Vgl. a. Schlieben-Lange 1983. 
und schwer kontrollierbare Kontextinformationen nur hinderlich seien für die Entdeckung der lokalen Verfahren. Als forschungspraktische Anleitung war die Dekontextualisierungsstrategie sehr wirkungsvoll, aber in ihrer Radikalität auf Dauer nicht durchzuhalten. Inzwischen hat sich ein sehr fruchtbarer Zusammenhang zwischen Gesprächsanalyse und Ethnographie der Kommunikation, der am Beginn der Konversationsanayse ausgeblendet wurde, hergestellt. ${ }^{14}$ Es bietet sich an, das hier existierende Wissen über Kommunikationsstrukturen in der Gesellschaft auch für Fragen der Zusammenstellung und Auswertung großer Text- und Gesprächskorpora nutzbar zu machen.

\section{Nächste Entwicklungsschritte in der autormatischen Verarbeitung gesprochener Sprache}

Ein direkter Zugriff auf gesprochene Sprache ohne Umwandlung in Symbolketten ist für die Auswertungszwecke der Linguistik wie der Informationsverarbeitung problematisch. Signalanalytische Verfahren werden für die Schnellerkennung von bestimmten Ereignissen (z.B. Sprache oder Nichtsprache, welche Sprache, d.h. die sog. »event detection«) und von Schlüsselwörtern (»key word spotting «) eingesetzt, um bei laufendem Ereignis schnell reagieren zu können (z.B. zu entscheiden, ob ein Kommunikationsvorgang aufgenommen wird oder nicht). Eine Schwäche dieses signalanalytischen Zugriffs ist, daß er mit keiner Semantik verknüpft ist und dementsprechend weitergehende informationsverarbeitenden Verfahren nicht greifen. Ein weiteres Problem ist die enorme Signaldatenmenge, der gegenüber die sprachliche Symboldarstellung eine starke Reduktion von Komplexität darstellt. ${ }^{15}$

Spracherkennung produziert Symbolketten. Die Hoffnung ist daher, daß Spracherkenner für die Verschriftung eingesetzt werden können. Alle heute vorliegenden Erkenner und Verfahren sind noch sehr beschränkt und stehen im Grunde erst am Anfang der Entwicklung zu einem sprecher- und bereichsunabhängigen Einsatz für natürlich gesprochene Sprache. Die schon relativ früh gehegte Hoffnung auf eine schnelle Entwicklung hat sich nicht erfüllt: "Even at that time [1961], I was assured that an automatic transcription of speech was just around the corner . It still is." (Sinclair 1991, S. 16).

Die Entwicklungsstrategien sind langfristig angelegt. Das muß auch für die Beteiligung der Gesprächsanalyse an diesem Komplex gelten. Damit wird

14 Davon zeugen allein im deutschen Sprachraum bereits eine ganze Reihe von ethnographisch-gesprächsanalytischen Arbeiten aus den letzten Jahren; vgl. dazu Kallmeyer (Hg.) 1994 und 1995.

15 Bei einer Abtastrate von 44 KH pro Sekunde und einer Erfassung jedes "Schnittes « mit 16 Bit ergibt das Informationsmenge von 704.000 Bit gegenüber etwa einem Wort, das als Symbolkette mit 8 Bit pro Buchstabe verarbeitet werden kann. 
zugleich aber auch wichtig, daß die schrittweise Entwicklung zu Zwischenergebnissen führt, die bereits nutzbringend für linguistische Zwecke eingesetzt werden können. Zur Illustration der Möglichkeiten kann das Projekts SERGES (Schriftliche ERfassung GEsprochener Sprache) beispielhaft skizziert werden, das zur Zeit am IDS in Zusammenarbeit mit dem Lehrstuhl Mustererkennung II der Universität Erlangen und weiteren Partner durchgeführt wird (vgl. Neumann et al. 1997). Ziel des Projekts ist ein Arbeitsplatz für computerunterstütztes Transkribieren und Analysieren von Gesprächsdaten. Zwei Arbeitsschwerpunkte sind:

- Die automatische Verknüpfung von Ton und Transkription (»alignment «) unter Einsatz eines Spracherkenners.

- Die Bildung von stochastischen Modellen über Transkriptionskorpora unter Nutzung der Recherche-Möglichkeiten des Datenbanksystems COSMAS (vgl. alWadi 1994) bzw. COSMAS II.

Im Rahmen des Projekts wird das gesprächsanalytische Korpus des IDS mit derzeit 180 transkribierten Aufnahmestunden als Ausstattung eines Testbetts für die Programmentwicklung zur Verfügung gestellt und nach Bedarf erweitert. Gegenwärtig ist nach der Digitalisierung des Tonkorpus und der Aufnahme der Transkripte in COSMAS II der nächste Arbeitsschritt die Vorbereitung der automatischen Text-Ton-Verknüpfung. Diese ist als erzwungene Zuordnung von Zeitsignalen zu vorhandenen Transkriptionen durchzuführen, wobei der Erkenner die von ihm produzierte Symbolkette mit der im Transkript vorgegebenen abgleicht. Im Prinzip ist der Einsatz eines Spracherkenners für die Text-Ton-Verknüpfung die gegenwärtig aussichtsreichste Verwendung. Die existierenden Spracherkenner haben allerdings große Schwierigkeiten mit fortlaufender, d.h. nicht wie beim abgehackten Sprechen in diskreten Einheiten produzierter Sprache sowie mit allen Eigenschaften der spontanen dialogischen Äußerungsproduktion wie Verschleifungen und Überlappungen. Die Aussicht auf Erfolg hängt vom Training des Spracherkenners $a b$, z.B. von dem ihm zur Verfügung stehenden Lexikon und den stochastischen Modellen, mit denen die Lexikoneinträge generiert sind. Das System muß die Eigenschaften der gesprochenen Sprache berücksichtigen ebenso wie Formen und Ausprägungen von Dialektalität im Sprachmaterial. Und so wie die Spracherkenner über ein Pausenmodell verfügen müssen (d.h. für Momente, in denen zwar Geräusche, aber keine sprachlichen Laute hörbar sind), benötigen sie auch ein Überlappungsmodell. Dieses gestattet ihnen, Beginn und Ende der Überlappungsstrecke dem Text zuzuordnen und ggf. die Überlappungsstrecke gar nicht oder mit anderen Verfahren zu verarbeiten. Die Leistung der automatischen Spracherkennung steigt mit der Zuverlässigkeit der vorab durchzuführenden Grobsegmentierung in relativ kleine und deutlich begrenzte Einheiten. Die Möglichkeit, nach einer Vorbereitung "von Hand« die Text-Ton-Verknüpfung für kleinere Ausschnitte des Materials automatisch durchführen zu können, stellt bereits ein wertvolles Zwischener- 
gebnis dar, das erhebliche Vorteile für die kombinierte Analyse von Transkript und Ton mit sich bringt. Wünschenswert und für die Anwendung des Systems auf große Datenmengen notwendig ist, daß die Grobsegmentierung »von Hand « ersetzt wird durch Verfahren der automatischen Segmentierung des Gesprächs in kleinere Einheiten als Vorsortierung für die Text-TonVerknüpfung. Zwei Gesichtspunkte erscheinen in diesem Zusammenhang wichtig:

(a) Der Einsatz von Verfahren der Sprecheridentifikation für die Erkennung von Sprecherwechsel. Bei geeigneten technischen Eigenschaften der Aufnahme gestattet das auch, die Sprecherbeiträge nach Kanälen zu trennen, was wiederum zu einer Erleichterung für den Spracherkenner führt; die Spuren werden anschließendwieder genau getaktet zusammengesetzt. Auf diese Weise kann einerseits die Worterkennungsrate gesteigert werden, andererseits sind die spezifischen Interaktionsbezüge für die Auswertung der Transkription wieder abgebildet. Sofern diese Bedingungen nicht erfüllt sind, muß mit dem vermischten Signal gearbeitet werden. Dementsprechend wird der Grobsegmentierer für die ursprünglichen Verhältnisse der Verzahnung von Sprechbeiträgen trainiert.

(b) Die statistisch basierte Entwicklung eines Sprachmodells für die Prosodie im Kontext von Segementierungsstellen (Binnensegmentierung komplexer Äußerungen, Äußerungsabschluß usw.). Hierbei wird auf der Grundlage eines hinreichend großen Korpus von transkribierten Gesprächsstücken mit einer Annotation der fraglichen Segmentierungsphänomene - eine Signalanalyse durchgeführt und mit einer statistischen Analyse ein Merkmalsprofil für bestimmte Segmentierungsarten errechnet. ${ }^{16}$ Die Kodierung enthält dabei interpretative Feststellungen über die Abschlossenheit oder Offenheit einer grammatischen Struktur, den Wechsel von Strukturebenen und die lokale Projektion der Äußerungsfortführung bzw. -beendigung. Das System setzt Verfahren der statistischen Analyse der Folge von Elementen aufeinander (nGramme) ein. Die Ergebnisse werden an der Kodierung als Zielvorgabe abgeglichen und nach Möglichkeit optimiert. Das "Training des Erkenners besteht darin, daß ihm die stochastischen Modelle für die beste Zielannäherung zur Verfügung gestellt werden (bzw. daß er sie in einem selbstlernenden Prozeß auswählt). In diesen Prozeß der Optimierung werden sukzessive unterschiedliche Komponenten einbezogen wie die Analyse von Wortkollokationen, die Prosodieanalyse und ein Tagging-Verfahren, das die Wortkette mit Wortklassen und morphologischen Eigenschaften annotiert.

Ein interessantes Problem, das über die Bedürfnisse der Grobsegmentierung hinausgeht und für die Erkennung von relevanten Kontexten bedeutsam ist, stellen Formen von Diskontinuität wie Parenthesen und Einschübe dar, d.h.

16 Für die prosodische Segmentierung von Dialogakten vgl. a. Batliner/Kompe/Kießling/Nöth/Niemann/Kilian 1995. 
Partnerinterventionen im Verlauf einer Äußerung. Das folgende Beispiel veranschaulicht eine interpretative Segmentierungsannotation für eine verschachtelte Parenthese. Die Form des einfachen Notationssystem ist relativ beliebig und ganz untechnisch (worauf es aber hier auch nicht ankommt): $[x]$ für den Beginn einer grammatischen Einheit, $[u / v]$ für grammatisch unvollständiges oder vollständiges Ende, $[o]$ für ein offenes Ende einer Äußerungssegments Fortsetzungsprojektion, $[e]$ und $[f]$ kennzeichnen Anfang und Ende eines Einschubs, wobei die Einbettungsebenen mit Zahlen angegeben werden. ${ }^{17}$

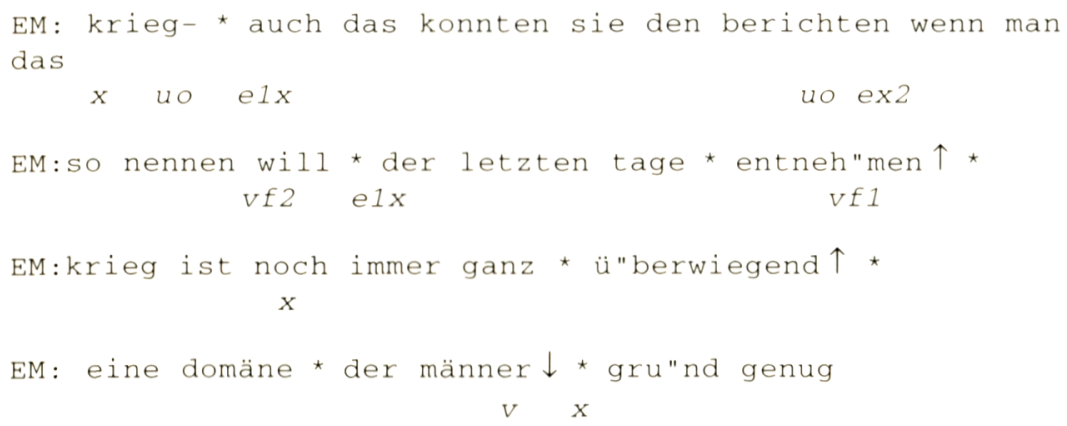

Notwendig ist die Erkennung der segmentierungsübergreifenden Bezüge von Formulierungseinheiten überall dort, wo definierte Kontexte eine Rolle spielen, z.B. für Kollokationsanalysen und grammatische Analysen. So zeigt [o] an, daß das Segment, an dessen Ende es erscheint, im Zusammenhang mit dem Folgesegment nach einem Einschub/einem Beitrag eines anderen Sprechers $\mathrm{zu}$ analysieren ist. Im Beispiel ist das für die übergeordnete grammatische Einheit (krieg ... krieg ist noch immer ganz überwiegend eine domäne der männer) aufgrund der Wiederholung von krieg kein so großes Problem; aber im Fall der Unterbrechung der Parenthese durch die Einbettung einer zweiten Parenthese wird eine Analyse ohne die Berücksichtigung der Einbettung von wenn man das so nennen will und den Anschluß von der letzten tage entnehmen an das Segment auch das konnten sie den berichten unmöglich. Wenn das Sprachverarbeitungssystem zumindest einige Eigenschaften derartiger Stellen kennt, kann es sie auch suchen. Damit wird eine wesentliche Verbesserung für den Zugriff auf große Korpora gesprochener Sprache geschaffen.

Das Problem, das sich mit Parenthesen in einer laufenden Äußerung stellt, verschärft sich mit Interventionen eines zweiten Sprechers (bzw. mehrerer weiterer Sprecher). Dialogisch sind gleichrangige und untergeordnete Redebeiträge unterschiedlicher Sprecher zu unterscheiden. Die damit zusammenhängenden Fragen sind im Laufe der 80er Jahre Forschungsgegenstand der Konversationsanalyse geworden. Zunächst, zur Zeit der Erarbeitung des Turn-taking-Modells, galt jede Intervention eines Beteiligten als Redebeitrag:

$17 \mathrm{Zu}$ den verwendeten Transkriptionszeichen vgl. die Erläuterung im Anhang. 
Rezeptionssignale ebenso wie Antworten auf Fragen oder Erzählankündigungen. In der Folge wurde eine Differenzierung des Rederechts bzw. des Geltungsstatus der Äußerung im interaktiven Austausch eingeführt. ${ }^{18}$ Daraus ergibt sich ein besonderes Interesse an Partnerinterventionen währende eines laufenden Redebeitrags des etablierten Sprechers. Dazu gehören Rückmeldungen wie $h m$ oder ja in allen Varianten, aber auch explizitere und umfangreichere Interventionen, die man unter der generellen Kategorie sEinwurf zusammenfassen kann (vgl. u.a. Fiehler 1985 u. Kotthoff 1993). Ein typischer Fall ist z.B.:

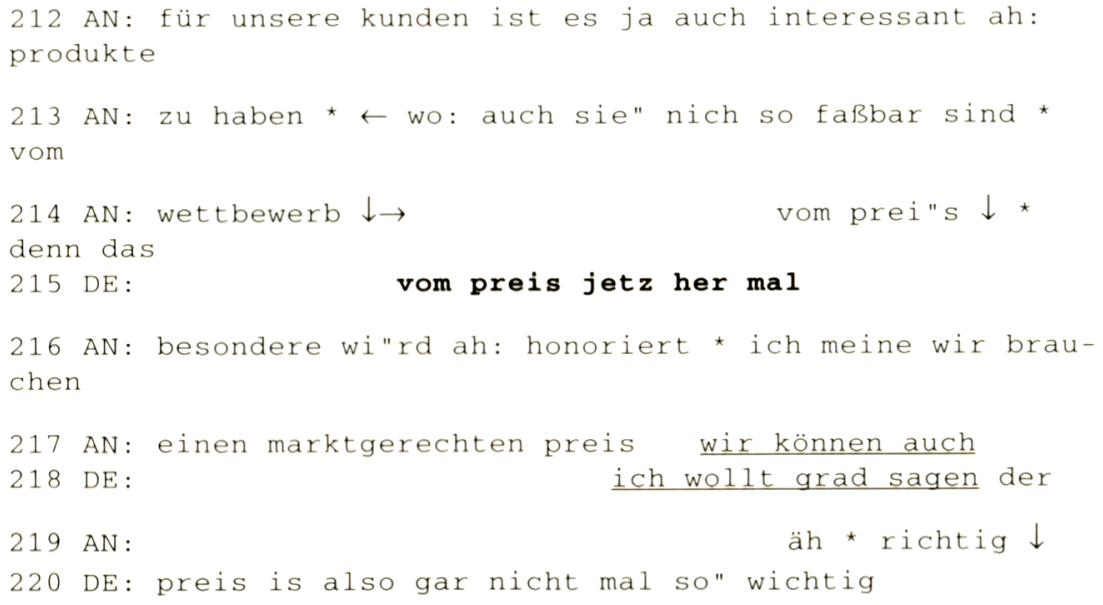

Hier sind eine Reihe von Eigenschaften des gemeinsamen Hervorbringens von Äußerungen in der Interaktion abzulesen. ${ }^{19}$ Eine Ausformulierung des linguistisch-gesprächsanalytischen Unterschungsprogramms zu solchen Phänomenen würde in etwa folgende Beobachtungspunkte enthalten:

(a) Initiative: Rezipienteninitiiert, ohne Einladung zur Intervention (bei weitem der häufigste Fall, so auch im Beispiel) oder sprecherinitiiert z.B. als Einladung zur Mitarbeit bei Formulierungsproblemen.

(b) Position: adjazent zu Bezugselement (unmittelbare Bezugnahme) und orientiert an Binnenzäszur (so im Beispiel). Die beiden Kriterien können unterschiedlich austariert werden. Häufig sind Verschiebungen des Einsatzpunktes (verzögerte und beschleunigte Adjazenz) unter Achtung/Mißachtung der Binnenstrukturierung (Zäsuren).

(c) Format: Für die Erkennbarkeit als Einwurf und damit als sekundärer

$18 \mathrm{Vgl}$. u.a. zusammenfassend Kallmeyer 1988.

19 Aus linguistischer Sicht ist das einer der interessantesten Gegenstände der Konversationsanalyse; vgl. u.a. Goodwin 1979. 
Beitrag zu einem laufenden primären Beitrag sind folgende Eigenschaften ausschlaggebend, welche die Unterordnung auf unterschiedlicher Ebene ausdrücken:

- Kürze (Interjektionen, elliptische Konstruktionen; bei vollständigen Sätzen Beschränkung auf die grammatisch notwendigen Satzglieder; nur geringfügige Expansionen im grammatischen Konstruktionsrahmen; keine komplexen Satzgefüge.

- Syntaktische Unvollständigkeit und Integration in die Konstruktion der Bezugsäußerung (Ellipsen, Einwort-Formulierungen, abhängige Phrasen, Nebensätze; so im Beispiel).

- Semantische und pragmatische Verknüpfung mit der Bezugsäußerung durch Proformen, Rededeixis, parallele Reformulierung.

(d) Funktion: Verständigungssicherung und Sachverhaltsklärung (Formulierungshilfe, Interpretationsexplizierung, Korrektur, Ergänzung, Präzisierung; vgl. Beispiel); Interaktionsmanagement (Steuerung von kommunikativen Handlungen, thematische Impulse); Bewertung (Kommentar zur Äußerung und zur Beteiligungsweise des primären Sprechers).

(e) Behandlung des Einwurfs durch den primären Sprecher: Typische Behandlungsformen sind: Integration (syntaktisch, prosodisch; so im Beispiel), Expandierte Behandlung als eigenständige Aktivität, Aufschieben und Übergehen.

(f) Folgeaktivitäten von primärem und sekundärem Sprecher: Fortsetzung des ursprünglichen Äußerungsplans; ggf. Nachbehandlung, z.B. als integrierte Berücksichtigung wie im Beispiel (AN verwendet preis erneut in seiner Fortsetzung) oder als Parenthese; erneute Intervention des Einwurf-Urhebers (wie im Beispiel) usw.

Die nur kurz skizzierte Art von Analyse zielt darauf, wesentliche Eigenschaften des interaktiven Zusammenspiel zu erfassen, d.h. vor allem die sprachlich-interaktiven Verfahren, mit denen die Sprecher das Verhältnis ihrer beiden Redebeiträge verdeutlichen und die Implikationen des Zwischenstatus eines sekundären Beitrags in Bezug auf die mit dem Rederecht verbundenen Aktivitätsmöglichkeiten ebenso wie die entsprechenden Implikationen für die weitere Wahrnehmung des Rederechts durch den primären Sprecher. ${ }^{20}$ Für das Training des Spracherkenners werden die Analysegesichtspunkte im gegenwärtigen Arbeitsschritt auf eine kleine Menge reduziert, die sich auf Position und Format des Einwurfs und den Beginn der Fortsetzung des primä-

20 Für die Analyse derartiger sprachlich-interaktiver Verfahren, ihre sequentiellen Implikationen und allgemein ihr rhetorisches Potential vgl. Kallmeyer/Schmitt 1996). 
ren Redebeitrags beziehen. Das Ergebnis der Erkennung wird im ersten Schritt noch wenig befriedigend sein. Einwürfe mit ihren spezifischen interaktiven Beziehungen können als ein paradigmatischer Fall für die Anforderungen an die Verarbeitung von dialogischer gesprochener Sprache angesehen werden. Gegenwärtig werden spontansprachliche Eigenschaften des Formulierens wie Anakoluthe, Pausen, Verzögerungen und Tempounterschiede verstärkt in die Entwicklung der Spracherkenner einbezogen. ${ }^{21}$ Die Berücksichtigung der interaktiven Verhältnisse macht jedoch noch wesentlich größere Schwierigkeiten und erfordert eine längerfristige Entwicklungsstrategie:

"We have a long way to go to reach entirely adequate models of human speech, but the foundation of all progress so far has been the careful fitting of appropriate models to large amounts of data (Libermann 1991b, 181).

Für die weitere Entwicklung der automatischen Erkennung von Einwürfen wird u.a. wichtig sein, Verfahren für die Lokalisierung des Bezugselements zu haben. Generell ist für die Erfassung der Bezüge zwischen den Formulierungen der Einsatz eines morphosyntaktischen Taggers notwendig. Bei der unmittelbaren Bezugnahme im Beispiel mit der Übernahme der syntaktischen Konstruktion des Bezugselements (vom [...]) im Einwurf und in der Fortsetzung (vom wetthewerb-vom preis jetzt her mal - vom preis) genügt schon eine einfache Kategoriserung der Nomen für eine starke Hypothese, daß es sich um Reformulierungen handelt. Aber auch bei weiter zurückgreifender Bezugnahme (verzögerter Adjazenz) wird zumindest ein Teil der Fälle zu verarbeiten sein:

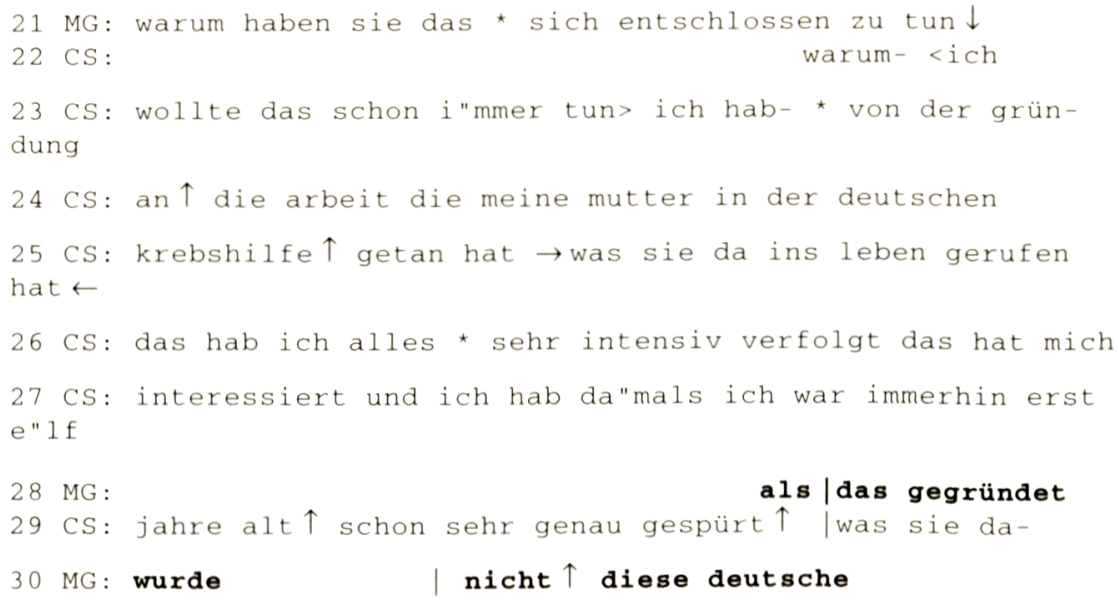

21 Für die Behandlung von Anakoluthen bei Reparaturen vgl. u.a. Tillmann/Tischer 1996). 


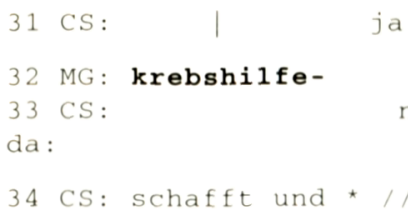

In diesem Beispiel ist damals (in Zeile 27) das letzte Temporaladverb in der Wortkette, und wenn für als im Einwurf eine temporale Lesart gefunden wird, kann nach einem geeigneten temporalen Bezugsausdruck gesucht werden. Fälle freier Reformulierung sind demgegenüber viel schwieriger und auf absehbare Zeit nicht zu lösen:

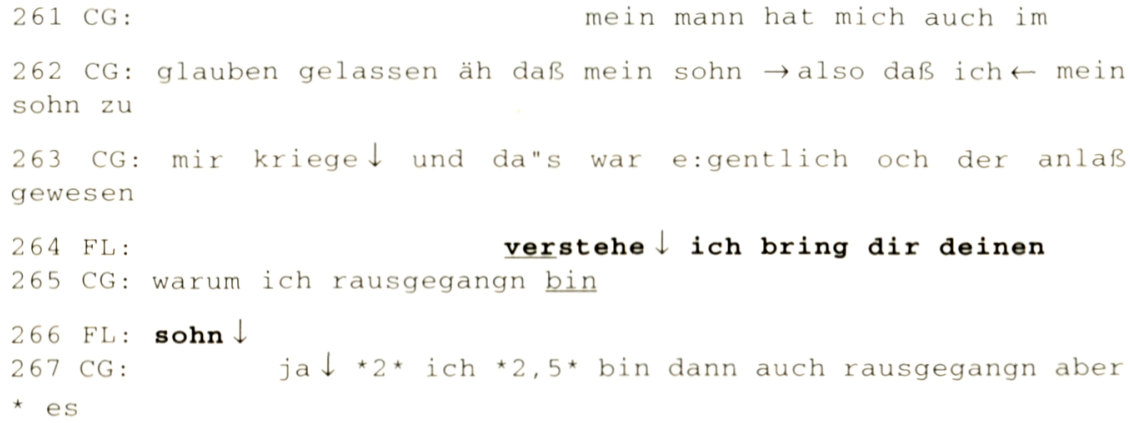

Um ich bring die deinen sohn als Reformulierung von mein mann hat mich auch im glauben gelassen daß ich mein sohn zu mir kriege zu erkennen, sind komplexe semantische Operationen unter Einbeziehung von Kontextwissen erforderlich. Eine Schlüsselinformation ist vermutlich, dass FL die Gesprächspartnerin CG im Kontext nicht duzt. Weiter muss eine semantische Beziehung zwischen jemanden im glauben lassen und einem Sprechakt, der als Rede wiedergegeben werden kann (»hat zu mir gesagt «), hergestellt werden. Um den Vorgang des Perspektivenwechsels bei der Reformulierung zu verstehen, sind noch eine Reihe weiterer Operationen notwendig, z.B. dass der Mann von CG (verdeckter) Agens von meinen sohn zu mir kriegen ist. ${ }^{22}$ Aber für den zunächst angestrebten Schritt der automatischen Verarbeitung, der auf die Auffindung von Einwürfen zielt, ist keine so tiefe Analyse notwendig.

Wenn das Sprachverarbeitungssystem aus der Menge der gefundenen Kandidaten für Einwürfe eine Reihe von Fällen mit einfachem Bezug zwischen Einwurf und Bezugselement angeben kann, ist schon sehr viel gewonnen.

Unter bestimmten Bedingungen liefert die Prosodie dazu Hilfen, wobei sich Fälle der unmittelbar adjazenten Plazierung als Kandidaten für die Ana-

22 Zu derartigen Aspekten der Kasusgrammatik vgl. Fillmore (1977) und Storrer (1996). 
lyse anbieten. Im Beispiel fällt neben der minimalen Übernahme der syntaktischen Konstruktion des Bezugselements (rom [...]) im Einwurf und in der Fortsetzung eine hochgradige prosodische Parallelität auf: Alle drei Formulierungseinheiten weisen eine Mikropause nach vom auf, die Akzentuierung des Nomens (mit einer schrittweisen Steigerung über die drei Formulierungseinheiten) und eine weitgehend identische Intonationsmuster mit einem terminalen Abfall (im Einwurf mit einer Streckung). Die Parallelität tritt besonders deutlich hervor, weil das Bezugselement bereits als eine Rechtsexpansion abgetrennt ist (wo: auch sie" nich so faßhar sind * vom wetthewerb $\downarrow$ )(vgl. a. Auer 1991) und mit einer markanten eigenständigen Intonationskontur realisiert wird. Erst bei der automatischen Prosodie-Analyse über einer hinreichend großen Fallmenge wird sich zeigen, ob es weitere, rekurrente und stabile prosodische Muster für unterschiedliche Typen von Einwürfen gibt. ${ }^{23}$

\section{Zum Einsatz von statistisch basierten Analyseverfahren}

Gegenwärtig zeichnet sich eine besondere Entwicklungsdynamik im Bereich der statistisch basierten Verfahren ab. ${ }^{24}$ Die Strategie der stochastischen Modellierung auf sehr großer Datengrundlage wird in der automatischen Sprachverarbeitung propagiert, weil es sich bei vielen der anstehenden Fragen anderen Verfahren der Extrapolation von Modellen/Regularitäten über kleinen Datenmengen überlegen zeigt (Libermann 1991). ${ }^{25}$ In diesem Kontext entwickeln sich auch Angebote von komplexen statistisch basierten Verfahren für die linguistische Sprachanalyse (vgl. oben Kap. 2).

Die Gesprächsanalyse hat sich bisher nur randseitig auf quantitative Untersuchungen eingelassen und nur in einer relativ einfachen Form. Für die Untersuchung der Beispielmengen genügte vielfach die Feststellung, daß »in der überwiegenden Mehrzahl der Fälle « eine Regularität zu beobachten ist und daß die abweichenden Fälle im Licht dieser Regulariät erklärbar sind. Die gängigen Verfahren der interpretativen Rekonstruktion funktionieren gut für die Entdeckung sehr allgemeiner, grundlegender »Methoden« der sprachlichen Interaktion. Erfolgreich war z.B. die Aufdeckung der sehr allgemeinen,

$23 \mathrm{Vgl}$. Mast/Kompe/Harbeck/Kießling/Niemann/Nöth/Schukat-Talamazzini/Warnke (1996) zum Versuch einer rein prosodischen Klassifikation von Dialogakten.

24 Für den Bereich der Spracherkennung vgl. u.a. Womser-Hacker 1992 und für das Taggen und die dabei anfallenden Aufgaben der Desambiguierung vgl. Wothke 1992.

25 Zumindest in Ansätzen scheint auch die bislang bestehende Konkurrenz zwischen regelbasierten (kognitiven) Verfahren und statistisch-basierten Verfahren in eine Verknüpfung beider Ansätze zu münden mit der Chance, die Stärken beider Seiten zum Tragen zu bringen. 
hochgradig rekurrent verwendeten und sehr stabil funktionierenden Verfahren des »turn taking « (Sacks/Schegloff/Jefferson 1974).

Schegloff (1993) formuliert deutlich seine Skepsis gegenüber dem Einsatz quantitativer Verfahren in der Konversationsanalyse. Sein zentrales Bedenken betrifft die Gefahr, daß die Reflexivität als grundlegende Eigenschaft der lokal wirksamen Herstellungsverfahren ausgeklammert wird. Die jeweilige sprachliche Aktivität stellt den für sie relevanten Kontext immer auch mit her, und die Bedeutung einer Äußerung ist Ergebnis des Interaktionsprozesses. Ob z.B. eine Äußerung ein Einwurf mit der Funktion der Verständigungssicherung ist, ggf. als Fremdkorrektur oder als bestätigungsheischende Verstehensexplizierung, kann erst entschieden werden, wenn die weitere Verarbeitung zeigt, daß der primäre Sprecher den Einwurft als solchen und nicht als eine um das primäre Rederecht konkurrierende Intervention behandelt. Bei einer quantitativen Untersuchung müssen die miteinander in Beziehung zu setzenden Elemente unabhängig voneinander erfaßt sein, z.B. Einwürfe unabhängig von den Eigenschaften der primären Äußerung und deren Fortsetzung. Manche konversationsanalytische Gegenstände wie die Formen des Sprecherwechsels sind inzwischen in ihren Normalformen und ihren speziellen Erscheinungsweisen unter ungewöhnlichen oder problematischen Bedingungen so präzise beschrieben, daß eine entsprechende Kodierung des Gesprächsmaterials möglich erscheint. Schegloff warnt aber generell davor, quantitative Untersuchungsverfahren vorschnell auf Gegenstände anzuwenden, deren Konstitutionsweise noch nicht hinreichend durch qualitative Analysen geklärt ist. Die Sorge ist dabei, daß vorausgesetzt wird, was eigentlich erst zu finden ist, und daß die heuristische Kraft des qualitativen Untersuchungsverfahrens verloren geht.

Heritage (1995) nimmt diese skeptische Position auf, relativiert sie aber (S. 404-406). Sinnvoll sei der Einsatz von quantitativen Verfahren für:

- Die Isolierung von »interessanten Phänomenen« (z.B. daß in Mehrpersonen-Gesprächen Lachen in typischer Weise sehr häufig anders inititiert wird als in Zweipersonengesprächen).

- Die Konsolidierung der Intuitionen in Bezug auf Gegenstände, bei denen eine große Menge von Fällen notwendig ist, um ein Verfahren zu erfassen (z.B. die »normale « maximale Länge von Gesprächspausen; der Anteil von Syntax, Semantik, Prosodie und Aktivitätsform an der Projektion der Beendigung eines Redebeitrags).

- Die indirekte Stützung eines Befundes (z.B. die Orientierung auf Vertraulichkeit durch den Zuschnitt von Redebeiträgen) durch unabhängige Beobachtungen (z.B. die Verwendung von solchen Formaten durch Familienmitglieder und gute Freunde).

- Das Aufzeigen des Zusammenhanges zwischen der Verwendung bestimmter Gesprächsverfahren und spezifischer sozialer oder psychologischer Kategorien (wie Geschlecht, Status usw.). 
Der erste und die beiden letzten Punkte enthalten im Kern klassische differentielle Fragestellungen, für die gängigerweise, z.B. auch in der Soziolinguistik, quantitative Verfahren eingesetzt werden. Der zweite Punkt enthält teilweise Fragestellungen mit umgekehrter Blickrichtung. Hier geht es z.B. darum, daß »in der überwiegenden Zahl der Fälle e ein Schwellenwert (maximale Pausenlänge) nicht überschritten wird; Oder das Problem liegt darin, daß eine allgemeine Regularität (Projektion des Äußerungsendes) wegen der hohen Varianz der Erscheinungsformen und komplexen wechselseitigen Abhängigkeit der Faktoren nur über große Mengen von Fällen erfaßbar ist. Beide Fragerichtungen die Entdeckung der allgemeinen Regularität unter der Fülle der Variation von Erscheinungsformen und die Entdeckung der Faktoren, mit denen die Varianz der Erscheinungsformen korrespondiert - sind geläufige Anwendungen quantitativer Methoden. Insofern ist plausibel, daß die konversationsanalytische Arbeit Nutzen ziehen kann aus der Methodenkombination. In den Ausführungen von Heritage wird deutlich, daß aus konversationsanalytischer Sicht ganz im Sinne des qualitativen Paradigmas der Sozialforschung (vgl. u.a. Glaser/Strauss 1967; Strauss 1991) - quantitative Verfahren vor allem zu heuristischen Zwecken, d.h. zur Hypothesengenerierung einschließlich der Hypothesenfestigung, aber nicht als Hypothesenüberprüfung in einem strengeren Sinne eingesetzt werden.

Die im Bereich der automatischen Sprachverarbeitung verwendeten statistischen Verfahren sind gegenüber den im Kontext der Gesprächsanalyse eingesetzten quantitativen Verfahren ungleich komplexer. Ein interessanter Berührungspunkt ist aber darin zu sehen, daß auch sie als heuristische Instrumente einsetzbar sind.

Belica (1996) skizziert unterschiedliche Strategien der Computerunterstützung mit aufsteigender Bedeutung der automatischen Analyse für das linguistische Ergebnis. Exemplifiziert werden die Vorgehensweisen anhand der Untersuchung von Neologismen anhand großer Korpora mit einer Zeitstruktur, d.h. Gliederung in Zeitsegmente, am Fall des sog. "Wendekorpus" des IDS mit Texten aus ost- und westdeutschen Zeitungen aus dem Zeitraum 1989-1993, zergliedert in extern (politisch)motivierte Zeitsegmente (Herberg/Steffens/Tellenbach 1997):

- Eine vorab festgelegte Hypothese wird geprüft, indem eine vom Rechner erstellte Synopse über den Sachverhalt (z.B. eine Beispielmenge mit Fundorten, etwa in einer zeitlich geordnetenListe) manuell analysiert und interpretiert wird.

- Eine sprachgebundene Hypothese wird in eine formelle mathematisch-

26 Das statistische Verfahren operiert mit den Schritten: Formulierung der NullHypothese entlang der Zeitachse $\left(\chi^{2}-\right.$ Text), Festlegung des Signifikanz-Niveaus, Überprüfung der Null-Hypothese entlang der Zeitachse. 
statistische Bedingung ohne Bezug auf konkrete Sprachelemente umformuliert. Zum Beispiel wird eine statistische Analyse und eine automatische Klassifikation von Kollokationen des Wortes Erneuerung veranlaßt.

- Das Programm errechnet ohne Vorgabe von Wörtern bestimmte Auffälligkeiten im Korpus und klassifiziert die Kollokationen. Die Anwendung des statistisch basierten automatischen Analyseverfahrens ${ }^{26}$ zeigt, daß interessante Wortkandidaten, deren Gebrauch sich signifikant verändert im betrachteten Zeitraum und die insofern als Schlüssel für den Wendediskurs und als Kandidaten für Sprachveränderung angesehen werden können, auch automatisch auffindbar sind.

Gerade die letzten, weitergehend automatisierten Verfahren können als ein heuristisches Instrument eingesetzt werden, um aus einem Korpus Phänomene auszufiltern, die nicht oder nur sehr schwer dinglich-konkret zu greifen sind und über die der Analysierende noch nichts wußte. Sie erfüllen insofern auf eine spezielle Weise die von Heritage angeführte Funktion, interessante Phänomene zu isolieren. Anders als die ersten beiden Verfahren hat das dritte nicht vom Linguisten wahrgenommene konkrete Sprachphänomene zum Ausgangspunkt, sondern abstrakte Relationen im Korpus. Die »selbstlernenden« Systeme der automatischen Sprachverarbeitung sind auf solche komplexabstrakten Hypothesenbildungen angewiesen.

Die sich hier abzeichnende Heuristik steht in krassem Gegensatz zur dezidiert konkreten, auf unmittelbare Wahrnehmbarkeit ausgerichteten, phänomenologisch fundierten Analysemethodik der Konversationsanalyse. Der durchschlagende Erfolg der Konversationsanalyse in den 70er Jahren basierte wesentlich darauf, daß regelhafte Zusammenhänge und Verfahren der elementaren Herstellung von sozialer Ordnung am Material sehr deutlich abzulesen sind. Die Leithypothese ist, daß die Interaktionsbeteiligten alles für die Definition der Situation und die Durchführung der Interaktion Relevante in hinreichender Weise für einander deutlich machen müssen. Die Aufzeigepraktiken der Beteiligten sind die Grundlage für die Rekonstruktion durch den Beobachter. Die Heuristik der Auseinandersetzung mit den "pieces of data « besteht gerade darin, sich durch das, was das Material »zeigt«, das am Material Sichtbare (ggf. Hörbare) anleiten zu lassen in der Hypothesenbildung über relevante Phänomene.

Die mathematische Abstraktion der statistischen Verfahren provoziert zwangsläufig die Angst des Gesprächsanalytikers, daß der mühsam etablierte Gegenstand »verbale Interaktion«, verstanden als konkret faßbarer Prozeß lokaler, situativer, zeitlich verlaufender Herstellung von Sinnstrukturen, wieder verlorengeht. Auf der anderen Seite sind die sich abzeichnenden Verfah-

27 Dabei ist Kollokation im allgemeinen Sinne von rekurrenten Wortfolgen zu verstehen, nicht im engeren phraseologischen Sinne. 
ren der Navigation in großen Korpora, der Recherche von Kandidaten für bestimmte Phänomene und der hypothesengesteuerten Korpuszusammenstellung so attraktiv als Unterstützung einer qualitativen Analyse, daß sich der Aufwand einer Beteiligung an der korpustechnologischen Entwicklung lohnt. Eine gewisse Beruhigung kann der Gesprächsanalytiker allemal daraus gewinnen, daß angesichts der Komplexität seines Gegenstandes die automatischen Verfahren auf absehbare Zeit nur eine subisidiäre Funktion im Bereich einer groben Vorsortierung haben können. Ein in absehbarer Zeit erreichbares Ziel ist z.B. ein statistisch fundiertes Kollokationen-Wörterbuch gesprochener Sprache ${ }^{27}$, das bei entsprechender Korpusgröße und -zusammensetzung ausdifferenziert werden kann nach Textsorten, Themen usw. Von Interesse wird dabei u.a. sein, Kontexteigenschaften wie Formen der Binnengliederung von Äußerungen, Sprecherwechsel und ggf. weitere sequentielle Strukturen einzubeziehen. Auf diese Weise sind z.B. schon Aufschlüsse über die Verwendung von sog. »Dialogwörtern/Geprächspartikeln«zu gewinnen. Die weitere Entwicklung wird zeigen, ob und wann komplexe Strukturen wie Einwürfe mit so großer Treffsicherheit automatisch gesucht werden können, daß das System über dem Korpus Auffälligkeiten der interaktiven Konstitution von Gesprächen errechnen und dem Gesprächsanalytiker zur Interpretation und weiteren Hypothesenbildung über die sprachliche Interaktion anbieten kann.

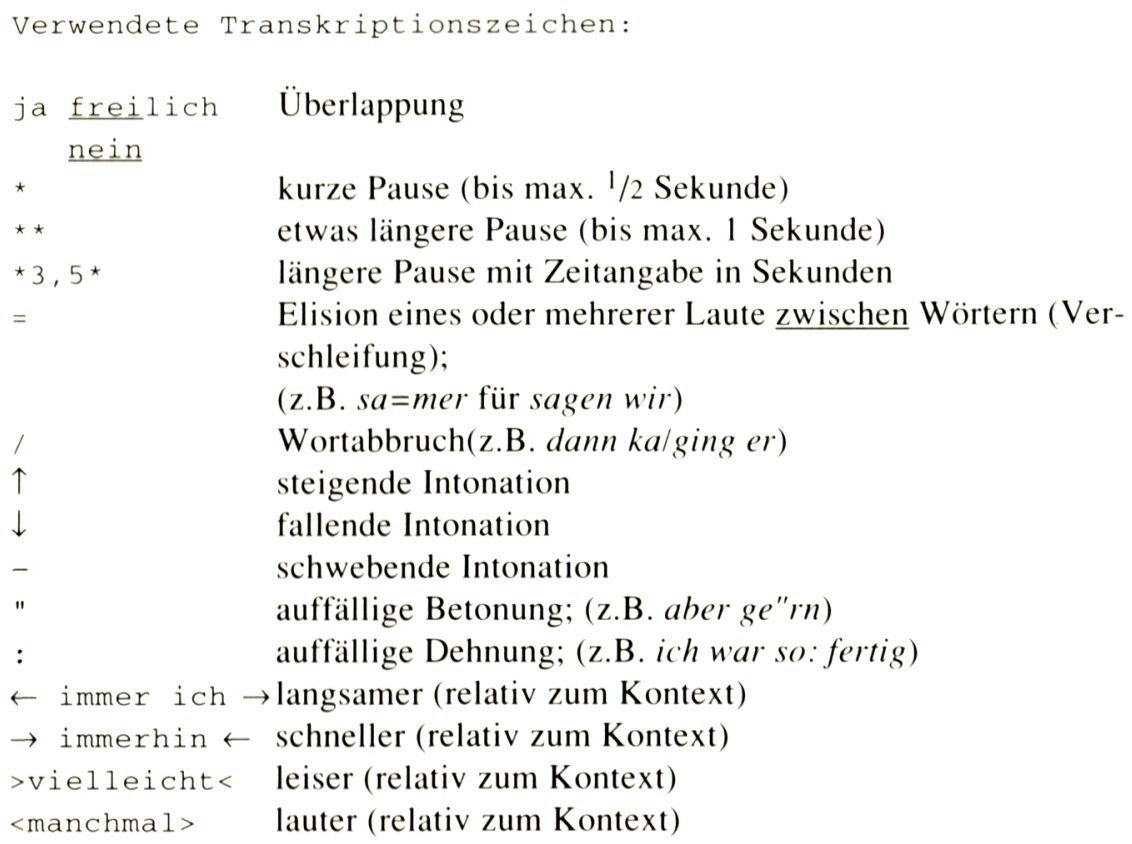


The advantage of technological change for linguistics:

Conversation analysis and automatic speech processing.

The author resumes new trends in speech technology and conversation analysis in the perspective of discovering points of common interest which could bring into contact the two quite distant and divergent worlds of research. The starting point is the demand for very large corpora of natural spoken dialogue in speech technology which could motivate new efforts of collecting corpora and storing them in a database format on the side of linguistic conversationalists. By participating in the development of corpus technology and the modelling of dialogical speech for the purposes of statistically based automatic procedures, linguists will get access to actually developing possibilities of retrieval and analysis which implies a new chance to answer already old questions about the nature of ongoing dialogical speech.

\section{Literatur}

Allen, Robert F. (1989): Survey of Natural Language Corpora in Computational Linguistics. In: Bátori/Lenders (eds.)(1989), 150-159.

al-Wadi, Doris (1994):COSMAS. Ein Computersystem für den Zugriff auf Textkorpora. Version R.1.3-1. Benutzerhandbuch. Institut für deutsche Sprache, Mannheim.

Auer, Peter (1991): Vom Ende deutscher Sätze. In: Zeitschrift für Germanistische Linguistik 19, S. 139-157.

Auer, Peter (1993): Über $\leftarrow$. In: Schlieben-Lange, Brigitte (Hg.): Materiale Bedingungen der Sprachwissenschaft. Zeitschrift für Literaturwissenschaft und Linguistik 90/91, 104-138.

Batliner, A./Kompe, R./Kießling, A./Nöth, E./Niemann, H./Kilian, U. (1995): The Prosodic Marking of Phrase Boundaries: Expectations and Results. In: RubioAyuso, Antonio J./López Solev, J. M. (eds.): Spreech Recognition and Coding New Advances and Trends. Berlin, 325-328.

Bátori, István S./Lenders, Winfried (eds.)(1989): Computational Linguistics. Computerlinguistik. An international Handbook on Computer Oriented Language Research and Applications. Ein internationales Handbuch zur computergestützten Sprachforschung und ihrer Anwendung. Berlin/New York 1989.

Belica, Cyril (1996): Statistische Analyse von Zeitstrukturen in Korpora. In: Neumann, Robert (Hg.): LDV-Info 8. Informationsschrift der Arbeitsstelle linguistische Datenverarbeitung. Institut für deutsche Sprache. Mannheim, S. 86-95.

Bergenholtz, Henning/Mugdan, Joachim (1989): Korpusproblematik in der Computerlinguistik: Konstruktionsprinzipien und Repräsentativität. In: Bátori/Lenders (Hg.), S. 141-149.

Bergenholtz, Henning/Schaeder, Burkhard (Hg.)(1979): Empirische Textwissenschaft. Probleme des Aufbaus und der Auswertung von Textkorpora. Königstein (Taunus).

Bungarten, Theo (1979): Das Korpus als empirische Grundlage in der Linguistik und Literaturwissenschaft. In Bergenholtz/Schaeder (Hg.)(1979), S. 28-51.

Dausendschön-Gay, Ulrich/Gülich, Elisabeth/Krafft, Ulrich (1992): Gemeinsam schrei- 
ben. Konversationelle Schreibinteraktionen zwihen deutschen und französischen Gesprächspartnern. In: Krings, Hans P./Antos, Gerd (Hg.), Texproduktion. Neue Wege der Forschung. Trier, S. 219-255.

Fiehler, Reinhard (1985): Einwürfe. In: Sucharowski, Wolfgang (Hg.), Gesprächsforschung im Vergleich. Analysen zur Bonner Runde nach der Hessenwahl 1982. Tübingen, S. 77-106.

Fillmore, Charles (1977): The case for case reopened. In: Heger, Klaus/Petöfi, János (Hg.), Kasustheorie, Klassifikation, semantische Interpretation. Hamburg, S. 326.

Galle, Matthias (1993): Methodische Grundlagen und formale Voraussetzungen der praktischen Auswertbarkeit sehr großer linguistischer Korpora (VCCL). In: Pütz, Horst P./Haller, Johannes (Hg.), Sprachtechnologie: Methoden, Werkzeuge, Perspektiven. Hildesheim, S. 246-261.

Glaser, Barney G./Strauss, Anselm L. (1967): The discovery of grounded theory. Strategies for qualitative research. London.

Gleason, H. A. (1955): An Introduction to Descriptive Linguistics. London.

Goodwin, Charles (1979): The interactive construction of a sentence in natural conversation. In: Psathas, George (ed.), Everyday Language: Studies in ethnomethodology. New York, S. 97-122.

Goody, Jack (1986): The logic of writing and the organization of the society. Cambridge.

Goody, Jack (1987): The interface between the oral and the written. Cambridge.

Günther, Hartmut/Otto, Ludwig et al. (Hg.) (1994/1996): Schrift und Schriftlichkeit. Ein interdisziplinäres Handbuch internationaler Forschung. Bd. 1. Berlin, New York 1994. Bd. 2. Berlin, New York 1966.

Haas, Walter/Wagener, Peter (Hg.)(1992): Gesamtkatalog der Tonaufnahmen des Deutschen Spracharchivs. 2 Bde. Tübingen (Phonai 38/39).

Herberg, Dieter/Steffens, Doris/Tellenbach, Elke (1997): Schlüsselwörter in der Wendezeit. Wörter-Buch zum öffentlichen Sprachgebrauch 1989/90. Berlin/New York.

Heritage, John (1995): Conversation Analysis: Methodological Aspects. In: Quasthoff, Uta (ed.), Aspects of Oral Communication. Berlin, S. 391-418.

Herzog, Marvin/Baviskar, Vera/Kiefer, Ulrike/Neumann, Robert/Putschke, Wolfgang/ Sunshine, Andrew/Weinreich, Uriel (eds.): The language and Culture Atlas of Ashkenazic Jewry. Bd. 1 Tübingen 1992, Bd. 2 Tübingen 1995.

Holly, Werner/Püschel, Ulrich (Hg.) (1993): Medienrezeption als Aneignung. Qualitative Medienforschung interdisziplinär. Opladen.

Humboldt, Wilhelm von (1827): Über den Dualis. Vortrag vor der Akademie der Wissenschaften am 26.4.1827. In: Humboldt, Wilhelm von, Schriften zur Sprachphilosophie. Darmstadt 1963, S. 113-143.

Kallmeyer, Werner (1988): Konversationsanalytische Beschreibung. In: Ammon, Ulrich/Dittmar, Norbert/Mattheier, Klaus J. (Hg.): Soziolinguistik. Ein internationales Handbuch zur Wissenschaft von Sprache und Gesellschaft. Bd. 2. Berlin, S. $1095-1108$.

Kallmeyer, Werner (1993): Wo bleibt der Kontext? Zur computerunterstützten Arbeit mit ethnographischen Korpora. In: Schlieben-Lange, Brigitte (Hg.): Materiale Bedingungungen der Sprachwissenschaft. Zeitschrift für Literaturwissenschaft und Linguistik 90/91, 88-103.

Kallmeyer, Werner (Hg.) (1994/1995): Kommunikation in der Stadt. Teil 1: Exempla- 
rische Analysen des Sprachverhaltens in Mannheim. Berlin, New York 1994. Teil 2: Ethnographien von Mannheimer Stadtteilen. Berlin, New York 1995.

Kallmeyer, Werner (i. Vorb.): Die Rezeption der soziologischen Konversationsanalyse und Ansätze zu einer linguistischen Gesprächsforschung. In: Auroux, Silvain/ Koerner, Konrad/Niederehe, Hans-Josef/Versteegh, Kees (eds.): Geschichte der Sprachwissenschaften. Ein internationales Handbuch zur Entwicklung der Sprachforchung. Berlin/New York.

Kallmeyer, Werner/Schmitt, Reinhold (1996): Forcieren oder: Die verschärfte Gangart. Zur Analyse von Kooperationsformen im Gespräch. In: Kallmeyer, Werner (Hg.), Gesprächsrhetorik. Rhetorische Verfahren im Gesprächsprozeß. Tübingen, S. $19-118$.

Klein, Ewan/Veltman, Frank (eds.)(1991): Natural Language and Speech. Symposium Proceedings, Brussels, November 26/27, 1991. Berlin/Heidelberg/New York.

Klein, Wolfgang (1985): Gesprochene Sprache - geschriebene Sprache. In: Zeitschrift für Literaturwissenschaft und Linguistik 59, S. 9-35.

Kotthoff, Helga (1993): Unterbrechungen, Überlappungen und andere Interventionen. Vorschläge zur Kategorienunterscheidung und kontextorientierten Interpretation. In: Deutsche Sprache 1993, 162-1985.

Liberman, Mark Y. (1991a): The Trend towards Statistical Models in Natural Language Processing. In: Klein/Veltman (eds.) (1991), S. 1-7.

Lieberman, Mark Y. (1991b): 5. The role of Linguistic Data in Speech Technology. In: Thomson (1991)(ed.), S. 179-182.

Mast, M./Kompe, R./Harbeck, S./Kießling, A./Niemann, H./Nöth, E./Schukat-Talamazzini, E.G./Warnke, V. (1996): Dialog act classification with the help of prosody. In: Proceedings of the International Conference on Spoken Language Processing, vol. 3. Philadelphia, S. 1728-1731.

Mehnert, Dieter (i. Vorb.): Historische Schallaufnahmen - Das Lautarchiv an der Humboldt-Uniersität zu Berlin. In: Studientexte zur Sprachkommunikation. Heft 13, Tagungsband zur 7. Konferenz Eletronische Sprachverarbeitung 25.-27. 11. 1996 in Berlin.

Neumann, Robert (1996): Korpora - Eine Herausforderung an die Informationserschließung. In: Feldweg, Helmut/Hinrichs, Erhard W. (Hg.): Lexikon und Text. Wiederverwendbare Methoden und Ressourcen zur linguistischen Erschließung des Deutschen. (Lexicographica, Series Maior 73). Tübingen, S. 13-22.

Neumann, Robert et al. (i. Vorb.): SERGES - Zur Integration gesprochener Sprache in korpuslinguistische Fragestellungen. In: Neumann, Robert (Hg.), LDV-Info 9. Informationsschrift der Arbeitsstelle Linguistische Datenverarbeitung. Institut für deutsche Sprache. Mannheim.

Olson, David R. (1994): The world on paper. The conceptional und cognitive implications of writing and reading. Cambridge.

Raumer, Rudolf von (1857): Offener Brief an den Herausgeber. In: Die deutschen Mundarten 4, S. 390-394.

Rieger, Burghard (1979): Repräsentativität. Von der Unangemessenheit eines Begriffs zur Kennzeichnung eines Problems linguistischer Korpusbildung. In: Bergenholtz/Schaeder (Hg.) (1979), S. 52-70.

Sacks, Harvey/Schegloff, Emanuel/Jefferson, Gail (1974): A simplest systematics for the organization of turn-taking for conversation. In: Language 50, S. 696735 . 
Schegloff, Emanuel A. (1993): Reflections on Quantification in the Study of Conversation. In: Research on Language and Social Interaction 26, S. 9-128.

Schlieben-Lange, Brigitte (1983): Traditionen des Sprechens. Elemente einer pragmatischen Sprachgeschichtsschreibung. Stuttgart.

Sinclair, John (1991): Corpus, Concordance, Collocation. Oxford.

Souter, Clive/Atwell, Eric (eds.)(1993): Corpus-based computational linguistics. Amsterdam/Atlanta.

Sperberg-McQueen, C. M./Burnard, Lou (eds.) (1994): Guidelines for Electronic Text Encoding and Interchange. TEI P3, Text Encoding Initiative. Chicago/Oxford.

Storrer, Angelika (1996): Verbbedeutung und Situationsperspektivierung. In: Grabowski, Joachim/Harras, Gisela/Herrmann, Theo (Hg.), Bedeutung, Konzepte. Bedeutungskonzepte. Opladen, S. 231-255.

Strauss, Anselm (1991): Grundlagen der qualitativen Sozialforschung. Datenanalyse und Theoriebildung in der empirischen soziologischen Forschung. München.

Thompson, Henry S. (ed.)(1991): Spoken Language Systems: Technological Goals and Integration Issues. In: Klein/Veltman (eds)(1991), S. 173-188.

Tillmann, Hans G./Tischer, Bernd (1996): Collection and Exploitation of Spontaneous Speech Produced in Negotiation Dialogues.

Wagener, Peter/Bausch, Karl-Heinz (i. Vorb): Tonaufnahmen des gesprochenen Deutsch. Dokumentation der Bestände von sprachwissenschaftlichen Forschungsprojekten und Archiven. Tübingen (Phonai 40).

Womser-Hacker, Christa (1992): Experimentelle Grundlagen und statistische Auswertung von Hypothesentests zur Mensch-Computer-Interaktion. In: Krause, Jürgen/Hitzenberger, Ludwig (Hg.).Computer Talk. Hildesheim, S. 84-104.

Wothke, Klaus (1992): Statistisch basiertes Worttagging von deutschen Textkorpora. Einige Experimente. In: Pütz, Horst/Haller, Johannes (Hg.), Sprachtechnologie. Hildesheim/Zürich/New York, S. 315-319. 